\title{
Structure and Mechanical Properties of Iron Subjected to Surface Severe Plastic Deformation by Attrition: II. Mechanical Properties of Nano- and Submicrocrystalline Iron
}

\author{
A. I. Yurkova ${ }^{a, b}$, Yu. V. Milman ${ }^{b}$, and A. V. Byakova ${ }^{a, b}$ \\ ${ }^{a}$ Kiev Polytechnical Institute, National Technical University of Ukraine, pr. Peremogi 37, Kiev, 03056 Ukraine \\ e-mail: yurkova@list.ru \\ ${ }^{b}$ Institute of Problems of Materials Science, National Academy of Sciences of Ukraine, Kiev, 03680 Ukraine \\ e-mail:milman@ipms.kiev.ua,byakova@mail.ru \\ Received July 15, 2008
}

\begin{abstract}
Depth-sensing indentation is used to study the effect of grain refinement to submicro- and nanograins on the mechanical properties (hardness, plasticity, Young's modulus) of armco iron subjected to severe plastic deformation by attrition in argon. In contrast to fcc metals, where the hardness increases and the plasticity decreases as the grain size decreases to $20 \mathrm{~nm}$, the hardness of bcc iron decreases from 5.8 to $3.7 \mathrm{GPa}$ and plasticity $\delta_{A}$ increases from 0.82 to 0.87 as the grain size decreases from 50 to $20 \mathrm{~nm}$.
\end{abstract}

DOI: $10.1134 / \mathrm{S} 0036029510040026$

\section{INTRODUCTION}

Grain refinement by severe plastic deformation (SPD) is one of the methods for improving the mechanical properties of structural materials [1]. It is known, however, that metals in a nanostructured state have a high yield strength and hardness and a low plasticity $[2,3]$. Therefore, when developing high-efficiency nanostructured materials, researchers should ensure an optimum relationship between the yield strength, which can be characterized by the hardness or nanohardness, and the plasticity. This means that the efforts made to increase the hardness are justified if the plasticity of the material remains satisfactory.

The study into the deformation behavior of nanomaterials is important due to the high structural sensitivity of the mechanical properties of a material. For example, the authors of [2-5] found that Young's modulus decreases with decreasing the grain size to submicro- and nanolevels as compared to conventional polycrystalline materials and that experimental curves deviate from the Hall-Petch relation, which implies a barrier action of grain boundaries (GBs) on dislocation motion. The Hall-Petch relation is usually valid for most nanomaterials to a certain critical grain size $d_{c}$, and the inverse effect (softening) is detected at a smaller grain size $\left(d<d_{c}\right)$.

The deviation from the Hall-Petch relation was thought to be related to a change in the plastic deformation mechanism in nanomaterials as compared to materials with a coarser grain structure [4, 6-9].

The properties of nanomaterials depend substantially on the structural-element size and the state of
GBs. Depending on the process of production, a grain structure with different degrees of structural inhomogeneity, misorientation, and imperfection and different chemical compositions of GBs can form. The authors of $[3-5,10-12]$ note that the anomalous mechanical behavior of nano- and submicrocrystalline materials depends mainly on GBs, near-boundary volumes, and triple junctions. This is especially true of the deformation structures produced by SPD methods: they have an imperfect structure of GBs caused by a high level of internal elastic stresses, a high grainboundary dislocation density, and significant microdistortions in a crystal lattice in near-boundary regions $[1,13-15]$.

The authors of [16] believe that, in contrast to coarse-grained materials where GBs serve as barriers to lattice dislocations, the GBs in nanocrystals can be considered as plastic-flow channels. Using molecular dynamics simulation, the authors of [4] showed that both grain-boundary sliding and another deformation mechanism, namely, intragrain sliding in which GBs serve as dislocation sources and sinks, are possible in nanocrystals. However, the latter mechanism has not been supported experimentally. We think that grainboundary sliding with an activated rotational deformation mode is the most probable plastic-deformation mechanism in nanomaterials at $d<d_{c}$.

Although the effect of the grain size on the mechanical properties of nanostructured fcc metals has been much studied [1-5], the data on the mechanical properties of nanostructured bcc metals, especially their plastic properties, are very scarce. The plasticity of bcc metals $(\mathrm{Cr}, \mathrm{Mo}, \mathrm{W})$ in the nanostruc- 
tured state during tension is such low that its values are usually not given [2]. However, the plasticity characteristic determined by nanoindentation makes it possible to estimate the plasticity of the materials that behave in a brittle manner during standard tensile tests.

The purpose of this work is to study the effect of the nanostructured state of iron produced by SPD by attrition (SPDA) in a neutral atmosphere (argon) on its mechanical properties (hardness, plasticity, Young's modulus).

\section{EXPERIMENTAL}

We studied armco iron (99.97 wt \%) samples with a gradient surface layer produced by SPDA in argon [17]; its grain structure changed from a micro- to submicro- and nanolevels [18].

The mechanical behavior of iron was analyzed by depth-sensing indentation using a Berkovich diamond pyramid and a Nano Indenter II device. Young's modulus $E$ and hardness $H_{h}$ were calculated using loading curves in the indenter load $F$-indenter displacement $h$ coordinates in terms of the Oliver-Pharr theory [19]. We determined Meyer hardness, which is the average contact pressure at the indenter-sample contact surface and correlates well with the flow stress [20]. During tests, we recorded the indenter displacement during both loading and unloading (Fig. 1).

The plasticity characteristic was determined using the procedure in [21-23] and calculated from the ratio of the areas in the indentation diagram,

$$
\delta_{A}=A_{p} / A_{t}=1-A_{e} / A_{t},
$$

where $A_{e}$ and $A_{t}$ are the areas under the unloading and loading curves, respectively, and $A_{p}=A_{t}-A_{e}$.

Plasticity characteristic $\delta_{A}$ determined by depthsensing indentation is an analog of dimensionless plasticity parameter $\delta_{H}$, which is the fraction of plastic deformation in the total elastoplastic deformation under an indenter. This parameter characterizes the formability of a material during deformation and is determined as

$$
\delta_{H}=\varepsilon_{p} / \varepsilon=1-\varepsilon_{e} / \varepsilon
$$

where $\varepsilon_{p}, \varepsilon_{e}$, and $\varepsilon$ are the plastic, elastic, and total strains along the loading direction averaged over the indenter-sample contact area, respectively.

It follows from Eq. (2) that plasticity parameter $\delta_{H}$ changes from 0 for absolutely elastic indentation to 1 for fully plastic deformation. In real practice, we have $0<\delta_{H}<1$, and the limiting cases are not detected in practice. The plasticity characteristic for metals and alloys that are plastic to failure under the conditions of standard tensile tests is $\delta_{H} \geq 0.9$ [22].

Although parameter $\delta_{H}$ can be determined by any mechanical test methods, it is better to determine it by nanoindentation. Plasticity characteristic $\delta_{H}$ depends

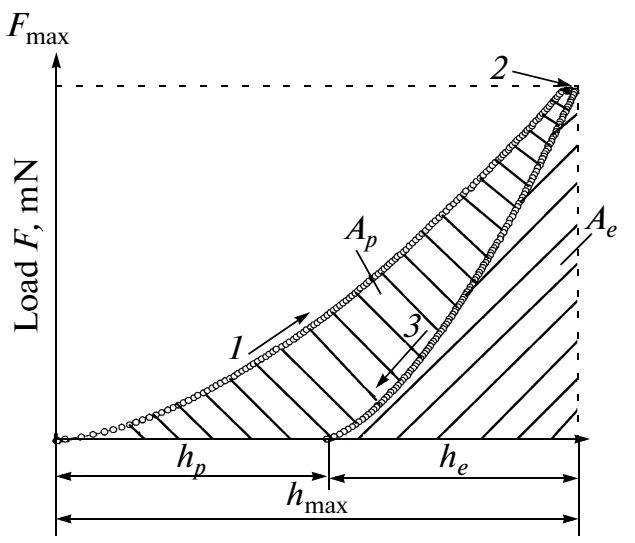

Fig. 1. Diagram of Berkovich indenter loading in the load $F$-displacement $h$ coordinates [20]: (1) loading of the indenter, (2) holding of the indenter at the maximum load, and (3) unloading of the indenter.

on total strain $\varepsilon$. However, during indentation by a pyramidal indenter, $\varepsilon$ is controlled by the indenter apex angle and, hence, is almost constant.

The plasticity characteristic can be calculated by the formula [23]

$$
\delta_{H}=1-\frac{H M}{E} \frac{\left(1-v-2 v^{2}\right)}{-\ln \sin \gamma},
$$

where $H M=F / S$ is the Meyer hardness, $v$ is the Poisson ratio of the material, and $\gamma$ is the angle between the pyramid axis and a pyramid face.

For a Berkovich indenter, plasticity characteristic $\delta_{H}$ is determined as

$$
\delta_{H}=1-10.2 \frac{H M}{E}\left(1-v-2 v^{2}\right) .
$$

For iron, $\delta_{H}$ was calculated from experimental data on $H M$ and $E$ at $v=0.27$ borrowed from [25].

The theoretical and experimental studies in $[24,26,27]$ showed that $\delta_{A} \approx \delta_{H}$ with a sufficient accuracy. As compared to $\delta_{H}$, plasticity characteristic $\delta_{A}$ is preferred, since the procedure of $\delta_{A}$ calculation, in contrast to the $\delta_{H}$ calculation, does not need the determination of Young's modulus, the hardness, and the Poisson ratio, which introduce certain errors.

\section{RESULTS OF NANOINDENTATION TESTS}

Figure 2 shows the indentation diagrams obtained for the undeformed coarse-grained base and the gradient surface layer of armco iron subjected to SPDA. The slopes of the unloading curves for the microcrystalline (curve 4), submicrocrystalline (curve 3), and nanostructured (curve 2, grain size is $50 \mathrm{~nm}$ ) states in the treated layer of iron are the same and identical to that of the coarse-grained base, and their $E$ moduli approximately correspond to the elastic modulus of the initial coarse-grained material. The slope of the 


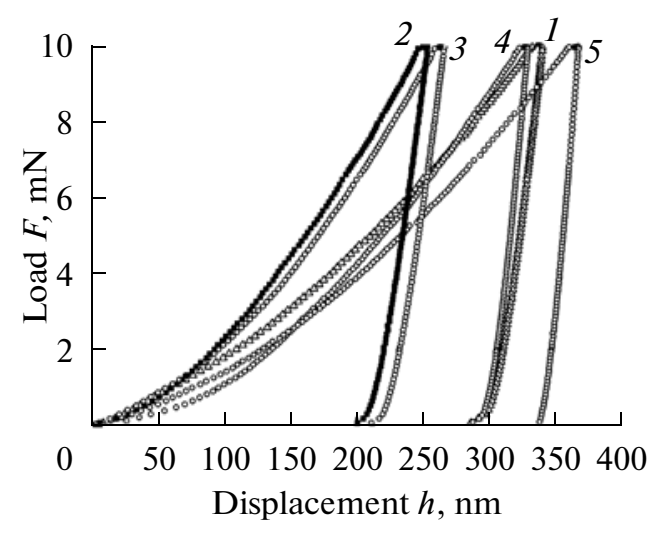

Fig. 2. Indentation diagram for a pyramid indenter penetrating into various regions of the surface layer of armco iron subjected to SPDA: (1) nanocrystalline region, $d=20 \mathrm{~nm}$ ( $\left.E=176 \mathrm{GPa}, H_{h}=3.7 \mathrm{GPa}\right) ;(2)$ nanocrystalline region, $d=50 \mathrm{~nm}\left(E=200 \mathrm{GPa}, H_{h}=5.9 \mathrm{GPa}\right) ;(3)$ submicrocrystalline region, $d=200 \mathrm{~nm}\left(E=209 \mathrm{GPa}, H_{h}=\right.$ $5.8 \mathrm{GPa})$; (4) microcrystalline region, $d=3 \mu \mathrm{m}(E=$ $212 \mathrm{GPa}, H_{h}=3.7 \mathrm{GPa}$ ); and (5) coarse-grained undeformed base, $d=80 \mu \mathrm{m}\left(E=210 \mathrm{GPa}, H_{h}=2.9 \mathrm{GPa}\right)$.

unloading curve for the nanostructured region next to the surface (curve 1), where the grain size is about $20 \mathrm{~nm}$, is slightly lower than that of the coarse-grained undeformed base (curve 5). This indicates a decrease in Young's modulus of nanocrystalline $\alpha$ as compared to the coarse-grained state. According to the data in Fig. 3, this decrease is about $10 \%$ (176 $\pm 9 \mathrm{GPa}$ against $210 \pm 10 \mathrm{GPa}$ ). It should be noted that Young's modulus of coarse-grained iron obtained by nanoindentation at room temperature falls in the $E$ range given in handbook [25] for coarse-grained $\alpha$ Fe (196.2-227.5 GPa).

Thus, SPDA in an argon atmosphere leads to a decrease in Young's modulus of iron only at a grain size smaller than $30 \mathrm{~nm}$; for larger grains (from nanoto submicro- and micrograins), the values of Young's modulus correspond to Young's modulus of coarsegrained iron with allowance for the experimental error (see Fig. 3).

The results of measuring iron hardness $H_{h}$ after SPDA demonstrate that it increases from 3 to $5.8 \mathrm{GPa}$ as the grain size decreases from coarse grains to submicron grains (to $200 \mathrm{~nm}$, Fig. 3). This effect is satisfactorily described by the Hall-Petch relation, $H=H_{0}+$ $k_{y} d^{-0.5}$, where $k_{y}=0.43 \mathrm{MPa} \mathrm{m}^{1 / 2}$, which almost coincides with this coefficient for ordinary polycrystalline iron $\left(k_{y}=0.39-0.73[28,29]\right)$. A further decrease in the grain size from 200 to $50 \mathrm{~nm}$ is accompanied by a deviation from the Hall-Petch relation: the hardness remains high and almost the same. A further decrease in the grain size from $50 \mathrm{~nm}$ causes a decrease in the hardness: in the region with a grain size of about $20 \mathrm{~nm}$, it is $3.7 \mathrm{GPa}$.

Plasticity characteristic $\delta_{A}$ in the attrition-refined layer as a function of the grain size reflects the hard-

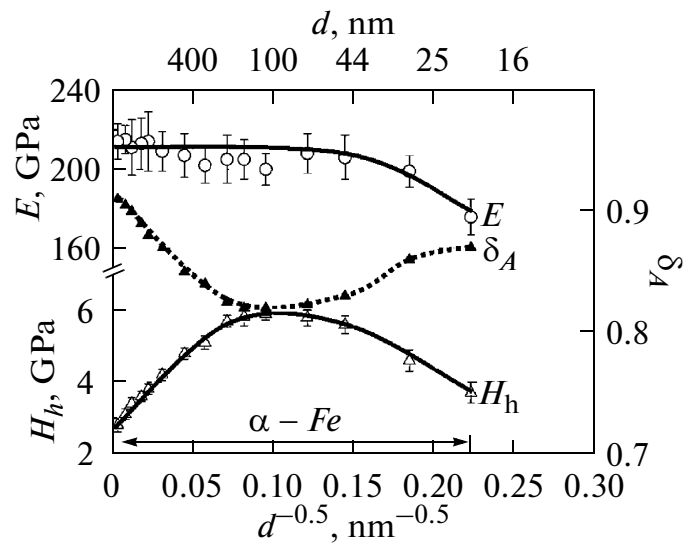

Fig. 3. Mechanical properties (hardness $H_{h}$, plasticity characteristic $\delta_{A}$, Young's modulus $E$ ) vs. grain size $d^{-0.5}$ of armco iron subjected to SPDA.

ness distribution (see Fig. 3). For example, the plasticity decreases with increasing hardness as the grain size decreases from coarse grains to submicro- and nanograins. When the grain size decreases to below $50 \mathrm{~nm}$, the hardness decreases and parameter $\delta_{A}$ increases from 0.82 to 0.87 .

\section{DISCUSSION OF RESULTS}

In $[1,2,4,15,16]$, several factors that affect the elastic properties of ultrafine-grained materials produced by SPD are discussed. GBs and triple junctions are assumed to play a key role in the decrease in the elastic properties of nanomaterials, since a significant fraction of atoms in a nanostructured material are located at sites that differ from the standard positions in a crystal lattice and the volume fraction of GBs, near-boundary volumes, and triple junctions increases as the grain size decreases $[3,10,12]$. According to the Mughrabi model for a composite [30, 31], Young's modulus $E$ can decrease as a result of an increase in the volume fraction of intergranular space, namely, GBs and triple junctions, whose mechanical properties differ from the properties of the grain body. Moreover, a decrease in the grain size leads to an increase in the fraction of free volume in GBs, near-boundary areas, and triple junctions. As a result, the interatomic bonds in a nanostructured material weaken.

According to the data in $[2,4]$, a deviation from the Hall-Petch relation for ultrafine-grained and nanocrystalline fcc metals is detected at a grain size $d \leq 20 \mathrm{~nm}$ : the hardness decreases slightly in this case (Fig. 4). However, for the bcc iron refined by SPDA (Fig. 3), a deviation from the Hall-Petch relation is detected for larger grains $(d \leq 200 \mathrm{~nm})$.

According to modern concepts, a deviation from the Hall-Petch relation is related to a change in the deformation mechanism, when an addition of grainboundary sliding (GBS) to the relay-race dislocation 


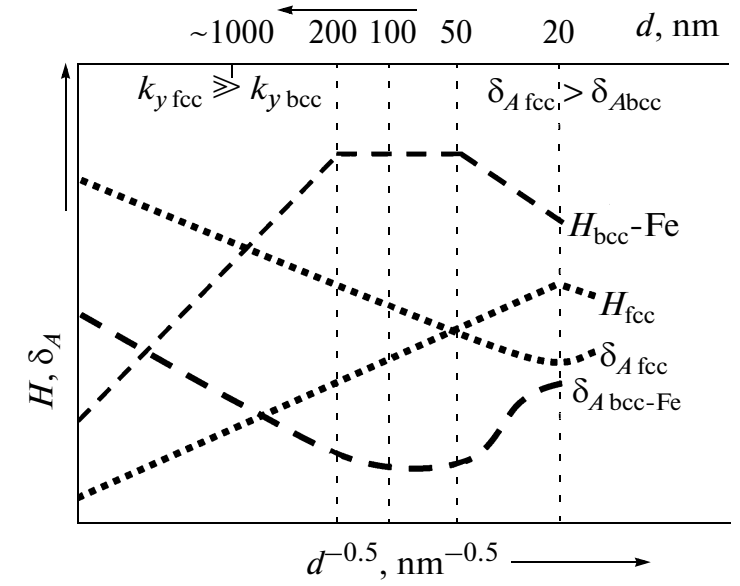

Fig. 4. Hardness $H$ and plasticity characteristic $\delta_{A}$ vs. grain size $d^{-0.5}$ of micro-, submicro-, and nanocrystalline fcc metals $[2,4]$ and bcc iron after SPDA.

transfer mechanism through GBs becomes energetically favorable. The values of coefficient $k_{y}$ in the Hall-Petch relation for bcc metals are significantly higher than those for fcc metals, $\sim 0.7$ and less than 0.16 , respectively [2]. According to [28, 29], $k_{y}=$ $0.39-0.73$ for iron, and $k_{y}=0.1$ for copper [4]. Therefore, as the strength increases sharply, the transfer of sliding from grain to grain requires very high stresses as the grain size decreases. As a result, a change in the deformation mechanism in bcc iron begins at a larger grain size. At a grain size of about $200 \mathrm{~nm}$ (against $20 \mathrm{~nm}$ in fcc metals), an addition of a GBS mechanism to the dislocation deformation mechanism in which sliding is transferred through GBs becomes energetically favorable. The former mechanism begins to play a key role at $d=50 \mathrm{~nm}$.

We used the differences between the hardnesses corresponding to the Hall-Petch relation and the experimental values for the nano- and submicrocrystalline layers $(20 \leq d \leq 200 \mathrm{~nm})$ and calculated the gain in the flow stress $\Delta \sigma_{S}$ (for $H \approx 3 \sigma_{S}$, according to Tabor [20]) upon a change in the deformation mechanism. With this quantity, one can estimate how GBS-assisted deformation is facilitated in comparison with the transfer of sliding from grain to grain through a grain boundary according to the Hall-Petch dislocation mechanism. For example, as the grain size decreases from 200 to $50 \mathrm{~nm}$, the gain in the flow stress $\Delta \sigma_{S}$ is $850 \mathrm{MPa}$, and we have $\Delta \sigma_{S}=2580 \mathrm{MPa}$ for a decrease to $d=20 \mathrm{~nm}$. These data demonstrate that, in the layer region with a grain size $50<d<200 \mathrm{~nm}$, deformation occurs via a mixed mechanism: the GBS mechanism is added to the conventional dislocation mechanism. At $d<50 \mathrm{~nm}$ (where the yield strength decreases), the GBS mechanism is dominant: rotational deformation modes are suppressed and rotational modes are activated [7]. In this case, deformation mainly proceeds

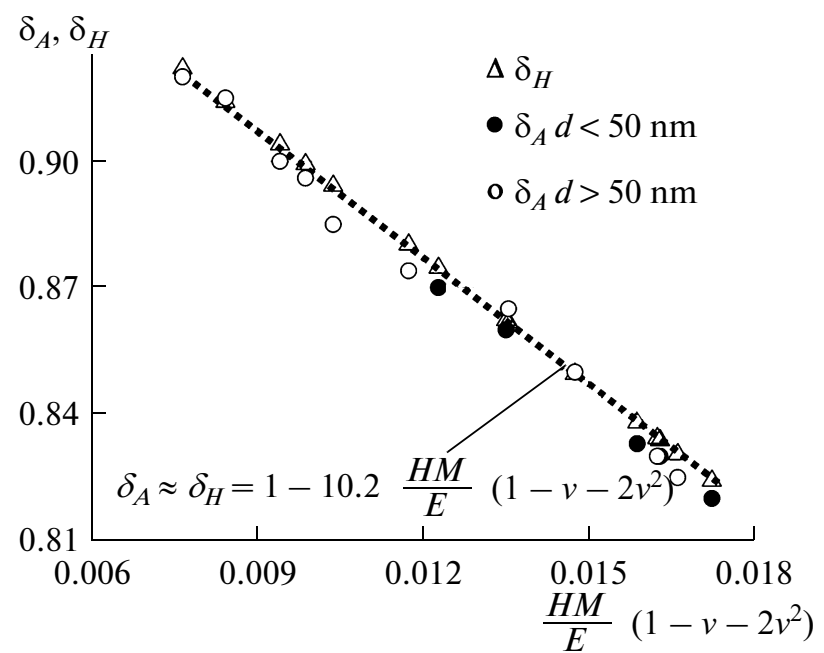

Fig. 5. Plasticity characteristics $\delta_{A}$ and $\delta_{H}$ of armco iron after SPDA vs. parameter $H M / E\left(1-v-2 v^{2}\right)$.

via nanograin rotation, which is caused by a high level of stresses in triple junctions.

Plasticity characteristics $\delta_{A}$ and $\delta_{H}$ calculated by Eqs. (1) and (3a), respectively, agree well with each other and exhibit a linear dependence on $H M / E\left(1-v-2 v^{2}\right)$, including the dependence for a nanostructured state (Fig. 5). The values of plasticity parameter $\delta_{A}$ fall on a straight line whose slope is $\tan \alpha=10.3$, which is close to the coefficient in Eq. (3a) for parameter $\delta_{H}$. This results supports the data in [24, 26, 27] indicating good agreement between parameters $\delta_{A}$ and $\delta_{H}$. Moreover, we experimentally confirmed the validity of the concepts following from the theory [22] that plasticity characteristic $\delta_{H}$ is mainly determined by the $H M / E$ ratio (see Eqs. (3), (3a)). Therefore, as the hardness increases, the plasticity characteristics decrease at a constant Young's modulus. In this case, the effect of a decrease in the plasticity with increasing hardness should be enhanced and $E$ should decrease.

It is interesting that plasticity characteristic $\delta_{A}$, which was calculated without using Young's modulus $E$, hardness $H M$, and Poisson ratio $v$, depends on the relationship between them and is described by Eq. (3a).

Despite a certain decrease in Young's modulus, the decrease in the hardness results in an increase in the plasticity characteristic (to 0.87 ) of nanostructured iron (see Fig. 3). The plasticity increases in the layer region with a grain size smaller than $50 \mathrm{~nm}$, since the hardness decreases faster than Young's modulus and the plasticity characteristic is mainly determined by the $H M / E$ ratio.

It is important that the grain refinement in bcc iron to a nanostructured state with a grain size smaller than $50 \mathrm{~nm}$ leads to an increase in plasticity characteristic $\delta_{A}$, whereas the grain refinement in fcc metals to a grain size of $20 \mathrm{~nm}$ leads only to a decrease in the plas- 
$d, \mathrm{~nm}$

$400.00100 .0044 .0025 .0016 .0011 .00 \quad 8.00 \quad 6.25$

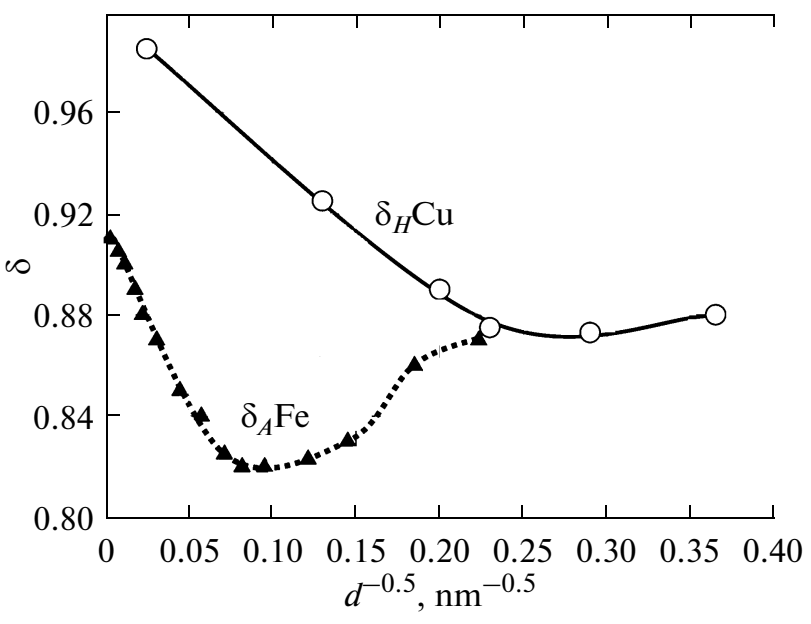

Fig. 6. Plasticity characteristics $\delta_{A}$ of iron after SPDA and $\delta_{H}$ of copper (calculated from $H\left(d^{-0.5}\right)$ [32]) vs. grain size $d^{-0.5}$.

ticity caused by an increase in the hardness. As follows from the data in Fig. 6, the plasticity characteristic of iron refined by SPDA to a grain size of $20 \mathrm{~nm}$ approaches the plasticity of copper with a grain size of $20 \mathrm{~nm}$ or smaller. It should be noted that it is very difficult to form a structure with a grain size $d<20 \mathrm{~nm}$, especially in engineering practice, and that the formation of nanograins with $d<50 \mathrm{~nm}$ in iron and the related increase in the plasticity can readily be achieved.

\section{CONCLUSIONS}

(1) As the grain size in armco iron decreases to below $30 \mathrm{~nm}$, Young's modulus decreases by $10 \%$ compared to the coarse-grained state $(176 \pm 9 \mathrm{GPa}$ against $210 \pm 10 \mathrm{GPa}$ ).

(2) As the grain size decreases from coarse grains to micron and submicron grains (to $200 \mathrm{~nm}$ ), the hardness increases from 3 to $5.8 \mathrm{GPa}$, which is satisfactorily described by the Hall-Petch relation. The value of coefficient $k_{y}$ in the Hall-Petch relation coincides with its value for coarse-grained iron. As the grain size decreases from 200 to $50 \mathrm{~nm}$, a deviation from the Hall-Petch relation is observed: the hardness is stabilized. A further decrease in the grain size from 50 to $20 \mathrm{~nm}$ results in a decrease in the hardness to $3.7 \mathrm{GPa}$ and an increase in plasticity characteristic $\delta_{A}$ from 0.82 to 0.87 . In fcc metals, a decrease in the grain size to $20 \mathrm{~nm}$ causes an increase in the hardness and a decrease in the plasticity, and the hardness decreases only at $d<20 \mathrm{~nm}$. These differences in the deformation behavior are related to a significantly higher coefficient $k_{y}$ in the Hall-Petch relation for bcc metals as compared to fcc metals.
(3) The plasticity characteristic of iron refined by SPDA to a grain size of $20 \mathrm{~nm}$ approaches the plasticity of copper with a grain size of $20 \mathrm{~nm}$ or smaller.

\section{ACKNOWLEDGMENTS}

We thank S.N. Dub for performing the depth-sensing indentation tests.

This work was supported in part by the National Academy of Sciences of Ukraine (project no. III-14-07) and the Ministry of Education and Science of Ukraine (project no. 2904-f).

\section{REFERENCES}

1. R. Z. Valiev and I. V. Aleksandrov, Nanostructured Materials Produced by Severe Plastic Deformation (Logos, Moscow, 2000) [in Russian].

2. N. I. Noskova and R. R. Mulyukov, Submicrocrystalline and Nanocrystalline Metals and Alloys (UrO RAN, Yekaterinburg, 2003) [in Russian].

3. R. A. Andrievskii and A. M. Glezer, "Size Effects in Nanocrystalline Materials: I. Structure Characteristics, Thermodynamics, Phase Equilibria, and Transport Phenomena," Fiz. Met. Metalloved. 88 (1), 50-73 (1999) [Phys. Met. Metallogr. 88 (1), 45-66 (1999)]; "II. Mechanical and Physical Properties," Fiz. Met. Metalloved. 89 (1), 91-112 (2000) [Phys. Met. Metallogr. 89 (1), 83-104 (2000)].

4. H. Van Swygenhoven and J. R. Weertman, "Deformation in Nanocrystalline Metals," Materialstoday 9 (5), 24-31 (2006).

5. K. S. Kumar, H. Van Swygenhoven, S. Suresh, "Mechanical Behavior of Nanocrystalline Metals and Alloys," Acta Materialia 51, 5743-5774 (2003).

6. V. A. Pozdnyakov and A. M. Glezer, "Structural Mechanisms of Plastic Deformation of Nanocrystalline Materials," Fiz. Tverd. Tela 44 (4) 705-710 (2002) [Phys. Solid State 44 (4), 600-605 (2002)].

7. N. I. Noskova, "Structural Features and Mechanisms of Deformation of Nanocrystalline Materials," J. Phys. Metals and Metallography 94 (Supplement), S119S130 (2002).

8. G. A. Malygin, "Violation of the Hall-Petch Law in Micro- and Nanocrystalline Materials," Fiz. Tverd. Tela 37 (8), 2281-2292 (1995).

9. V. A. Pozdnyakov, "Mechanisms of Plastic Deformation and the Anomalies of the Hall-Petch Dependence in Metallic Nanocrystalline Materials," Fiz. Met. Metalloved. 96 (1), 114-128 (2003) [Phys. Met. Metallogr. 96, 105-119(2003)].

10. A. M. Glezer, "Strength and Plasticity of Nanocrystals," in Proceedings of the Ist International Conference on Deformation and Fracture of Materials, Moscow, Russia (Moscow, 2006), pp. 14-16.

11. M. Yu. Gutkin and I. A. Ovid'ko, Defects and Plasticity Mechanisms in Nanostructured and Noncrystalline Materials (Yanus, St. Petersburg, 2001) [in Russian].

12. G. Palumbo, U. Erb, and K. T. Aust, "Triple Line Dislocation Effects on the Mechanical Behavior of Materials,” Scr. Metall. Mater. 24, 2347-2350 (1990). 
13. R. Z. Valiev and R. Sh. Musalimov, "High-Resolution Transmission Electron Microscopy of Nanocrystalline Materials," Fiz. Met. Metalloved. 78 (6), 114-121 (1994) [Phys. Met. Metallogr. 78 (6) 666-670 (1994)].

14. R. Z. Valiev and R. K. Islamgaliev, "Structure and Mechanical Behavior of Ultrafine-Grained Metals and Alloys Subjected to Intense Plastic Deformation," Fiz. Met. Metalloved. 85 (3), 161-177 (1998) [Phys. Met. Metallogr. 85 (3), 367-377 (1998)].

15. Z. Horita, D. Smith, M. Furakawa, et al., "An Investigation of Grain Boundaries in SubmicrometerGrained Al-Mg Solid Solution Alloys Using HighResolution Electron Microscopy," J. Mater. Res. 11 (8), 1880-1890 (1996).

16. M. Yu. Gutkin and I. A. Ovid'ko, Physical Mechanics of Deformed Nanostructures. Vol. I. Nanocrystalline Materials (Yanus, St. Petersburg, 2003) [in Russian].

17. A. I. Yurkova, A. V. Belotskii, Yu. V. Milman, and A. V. Byakova, "Nanostructure Formation on the Iron Surface during Attrition," Nanofizika, Nanosistemy, Nanomaterialy 2 (2), 633-644 (2004).

18. A. I. Yurkova, Yu. V. Milman, and A. V. Byakova, "Structure and Mechanical Properties of Iron after Surface Severe Plastic Deformation by Attrition: I. Structure Formation," Deformatsiya Razrushenie Materialov, No. 1, 2-12 (2009).

19. W. C. Oliver and G. M. Pharr, "An Improved Technique for Determining Hardness and Elastic Modulus Using Load and Displacement Sensing Indentation Experiments," J. Mater. Res. 7 (6), 1564-1583 (1992).

20. D. Tabor, The Hardness of Metals (Clarendon, Oxford, 2000).

21. Int. Standard ISO 14577-1-2002 (E).

22. Yu. V. Milman, B. A. Galanov, and S. T. Chugunova, "Plasticity Characteristics Obtained through Hardness Measurement," Acta Metal. Mater. 41 (9), 2523-2532 (1993).
23. Yu. V. Milman, S. T. Chugunova, and I. V. Goncharova, "Plasticity Characteristic Obtained by Indentation Technique for Crystalline and Noncrystalline Materials in Wide Temperature Range," High Temperature Materials and Processes 25 (1-2), 39-46 (2006).

24. A. Byakova, Yu. V. Milman, and A. Vlasov, "High Performance Ceramic Coatings for Cutting Tool-Perspective in Improvement of Coating Mechanical Properties," in Proceedings of the 8th CIRP iNternational Workshop on Modeling of Machining Operations, Chemnitz, Germany (Chemnitz, 2005), pp. 559-568.

25. I. N. Frantsevich, F. F. Voronov, and S. A. Bakuta, Elastic Constants and Elastic Moduli of Metals and Nonmetals: A Handbook (Naukova Dumka, Kiev, 1982) [in Russian].

26. Yu. V. Milman, "Plasticity Characteristic Obtained by Indentation," J. Phys. D: Appl. Phys. 41, 1-9 (2008).

27. Yu. Milman, S. Dub, and A. Golubenko, "Plasticity Characteristic Obtained through Instrumental Indentation," Mater. Res. Soc. Symp. Proc. 1049, 123-128 (2008).

28. V. I. Trefilov, V. F. Moiseev, E. P. Pechkovskii, et al., Strain Hardening and Fracture of Polycrystalline Materials (Naukova Dumka, Kiev, 1987) [in Russian].

29. V. I. Trefilov, Yu. V. Milman, and S. A. Firstov, Physical Foundations of the Strength of Refractory Materials (Naukova Dumka, Kiev, 1975) [in Russian].

30. T. Ungar, H. Mughraby, D. Könnpagel, and M. Wilkens, "Line-Broadening Study of the Dislocation Cell Structure in Deformed [001]-Oriented Copper Single Crystals," Acta Metall. 32 (3), 333-342 (1984).

31. H. Mughraby, "Dislocation Wall and Cell Structures and Long-Range Internal Stresses in Deformed Metal Crystals," Acta Metall. 31, 1367-1379 (1983).

32. S. Cheng, E. Ma, M. Y. Wang, et al., "Tensile Properties of In Situ Consolidated Nanocrystalline Cu," Acta Materialia 53, 1521-1533 (2005). 